\title{
Handheld Micromanipulation with Vision-Based Virtual Fixtures
}

\author{
Brian C. Becker, Student Member, IEEE, Robert A. MacLachlan, Member, IEEE, Gregory D. Hager, \\ Fellow, IEEE, Cameron N. Riviere, Member, IEEE
}

\begin{abstract}
Precise movement during micromanipulation becomes difficult in submillimeter workspaces, largely due to the destabilizing influence of tremor. Robotic aid combined with filtering techniques that suppress tremor frequency bands increases performance; however, if knowledge of the operator's goals is available, virtual fixtures have been shown to greatly improve micromanipulator precision. In this paper, we derive a control law for position-based virtual fixtures within the framework of an active handheld micromanipulator, where the fixtures are generated in real-time from microscope video. Additionally, we develop motion scaling behavior centered on virtual fixtures as a simple and direct extension to our formulation. We demonstrate that hard and soft (motionscaled) virtual fixtures outperform state-of-the-art tremor cancellation performance on a set of artificial but medically relevant tasks: holding, move-and-hold, curve tracing, and volume restriction.
\end{abstract}

\section{INTRODUCTION}

$I^{N}$ biology and microsurgery, proper manipulation of extremely small anatomical features often requires precision and dexterity exceeding the capability of the unaided operator. Physiological tremor, high-frequency involuntary hand movement with amplitudes of over $100 \mu \mathrm{m}$ [2], is a large contributing factor to the difficulty of micromanipulation. Furthermore, lower frequency drift in gross hand positioning over time is also problematic [3] and is often due to weak depth perception through the microscope and the relatively low-bandwidth eye-hand feedback loop. Various robotic platforms such as the Robot Assisted MicroSurgery (RAMS) system [4] and the Johns Hopkins SteadyHand [5] have been proposed to deal with these problems in micromanipulation. In general, robotic control aids for micromanipulation can be grouped into three categories: tremor compensation, motion scaling, and virtual fixtures.

Tremor compensation is a key component of many surgical robots $[5,6]$ and tries to eliminate frequency bands dominated by tremor in order to remove unwanted motion while preserving the operator's intended movements. In cooperative robots such as SteadyHand, this can be done

Manuscript received September 15, 2010. This work was supported in part by the National Institutes of Health (grant nos. R01 EB000526, R21 EY016359, and R01 EB007969), the American Society for Laser Medicine and Surgery, the National Science Foundation (Graduate Research Fellowship), and the ARCS Foundation.

B. C. Becker, R. A. MacLachlan, and C. N. Riviere are with the Robotics Institute, Carnegie Mellon University, Pittsburgh, PA 15213 USA (e-mail: camr@ri.cmu.edu).

G. D. Hager is with the Computer Science Department, Johns Hopkins University, Baltimore, Maryland 21218 USA. mechanically by utilizing the inherent stiffness of the robot arm to dampen high-frequency movement. In robots where the actuation is independent of the hand movement, tremor compensation can be achieved by inserting filters between the hand motion and the drive mechanism to reduce the higher-frequency tremor while retaining the operator's gross lower-frequency movements.

Motion scaling, often used in conjunction with tremor suppression, scales movements so the tip only moves a set fraction of the hand movement. For example, a scale factor of $1 / 2$ would transform hand movement of $2 \mathrm{~mm}$ to $1-\mathrm{mm}$ movement of the robotic tip. Recent developments in tremor compensation describe a low-pass shelving filter [1], which acts as a hybrid tremor suppression filter that affords relative motion scaling and reports an additional $30-50 \%$ reduction in tremor compared to a low-pass filter.

Virtual fixtures, in contrast to tremor compensation or motion scaling that operate in the general case, instead aim to improve specific motions or tasks [7]. Much like a ruler aids in the specific instance of drawing straight lines, virtual fixtures can be thought of as software guides that constrain the robot motion in specific ways. Most prominently, the SteadyHand robot has reported success in using virtual fixtures in several applications [8, 9].

A fully handheld micromanipulator called Micron [1] has been developed with actuators not on a robot arm, but between the handle grip and the tool tip. Micron has a roughly $2 \times 2 \times 1 \mathrm{~mm}$ range of motion in which it can move the tool tip independently of the hand motion, allowing it to perform behaviors such as tremor compensation, motion scaling, and virtual fixtures. In the remainder of this paper, we derive a simple position-based virtual fixture framework for handheld micromanipulators such as Micron that incorporates both tremor compensation and motion scaling. Section II covers background material and introduces Micron and the system setup. Section III presents our derivation of virtual fixtures for Micron. In Section IV, we

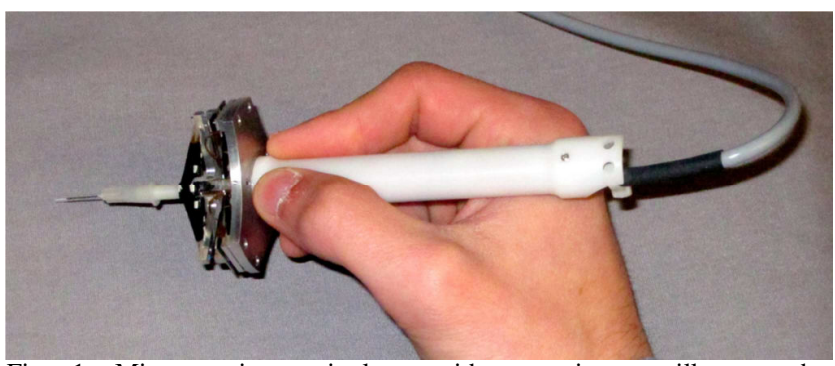

Fig. 1. Micron micromanipulator without casing to illustrate the piezoelectric motors between the handle and tip of the instrument, which enable the tip to actuate independently of the handle (or hand) motion. 
test our system with medically relevant experiments. Finally, we conclude in Section $\mathrm{V}$ with a discussion of results and directions for future work.

\section{BACKGROUND}

A significant amount of research has been accomplished in applying virtual fixtures to various robotic platforms and micromanipulation problems $[7,10,11]$. In most formulations of virtual fixtures, the user manipulates a robot arm that is attached to the instrument directly or remotely via tele-operation. Forces on the robot arm are transformed to velocity commands at the instrument tip. These velocities are shaped first in software via constraints as specified by the active virtual fixture. If the robot is non-backdriveable, strict adherence to the virtual fixture can be enforced by ensuring zero velocity components in directions that move the tip away from the fixture.

Unlike most existing virtual fixture enabled robots, Micron is not manipulated by the operator through the application of forces to a joystick control or robot arm. It is a fully handheld device that purely senses positions; thus, the input to the virtual fixtures must be handle (or hand) motion. This fundamental difference, the use of position instead of force as the control input, necessitates the development of a different formulation of virtual fixtures specifically designed for this class of handheld micromanipulators.

\section{A. System Setup}

The handheld system developed in our lab is Micron, a previously reported 3 DOF micromanipulator [1]. Unlike a robotic arm such as the SteadyHand, Micron is fully handheld and has motors positioned between the handle grip and the instrument tip as depicted in Figure 1. The motors are three Thunder ${ }^{\circledR}$ piezoelectric actuators arranged in a star pattern, allowing for tip movement independent of hand motion within an approximate $2 \times 2 \times 1-\mathrm{mm}$ range of motion centered on the handle.

Low-latency, high-bandwidth positioning information is obtained from custom optical tracking hardware named ASAP [12]. Three LEDs on the actuated shaft of the instrument allow for 6 DOF position tracking of Micron's tip. An additional LED attached to the handle provides hand movement information. Position sensitive detectors (PSDs) detect the LEDs within a $4 \mathrm{~cm}^{3}$ workspace at a rate of $2 \mathrm{kHz}$, with measurement accuracies of $<10 \mu \mathrm{m}$ RMS.

The operator uses Micron under a 29X surgical microscope (Zeiss OPMI 1). A 27 gauge hypodermic needle with a $400 \mu \mathrm{m}$ shaft diameter is attached to Micron with a 5 mil Nitinol wire inserted into the needle to serve as a very fine instrument tip. The Nitinol wire is tapered to a blunt point and painted white for enhanced contrast. Stereo cameras (PointGrey Flea2) attached to the microscope view the same workspace as the operator and capture video at 54 $\mathrm{Hz}$ with a custom resolution of $504 \times 324$. The cameras track both the tip of the instrument and anatomical targets. The cameras are registered to ASAP so visual information

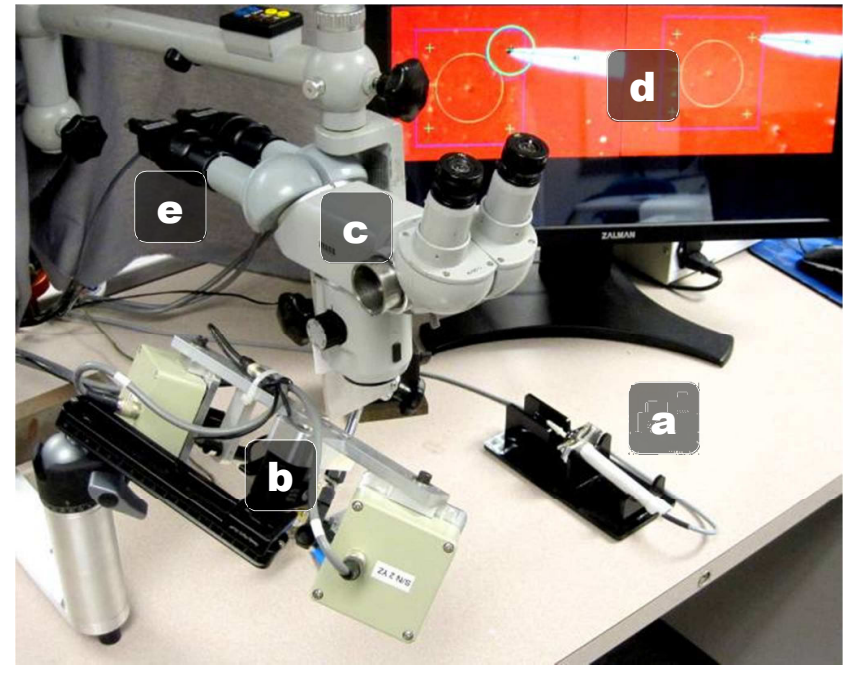

Fig. 2. System setup with (a) Micron, (b) ASAP position sensors, (c) surgical microscope, (d) 3D monitor, and (e) stereo cameras.

regarding Micron's tip and tracked targets can be used for control [13]. The entire setup can be seen in Figure 2.

\section{B. Problem Definition}

Represented as a subspace defined in 3D Euclidean space by the stereo vision cameras, the virtual fixture must constrain the tip position of Micron, using only the hand motion of the operator to guide and position the instrument tip on the virtual fixture. In the case of hard virtual fixtures, the tip of Micron should always lie on the subspace; in the case of soft virtual fixtures, the error between the hand motion and the virtual fixture should be scaled. In both cases, tremor compensation should smooth tip movement.

\section{METHODS}

We denote the tip of Micron in 3D space as $P_{T} \in \mathbb{R}^{3}$. As Micron actuates, the tip will move independently of the hand (or handle) motion. We define the null position as the 3D tip position $P_{N} \in \mathbb{R}^{3}$ under the assumption that Micron is off; in another words $P_{N}$ exactly reflects the hand motion. One can think of $P_{N}$ as being mechanically tied to the handle, and unless Micron actuates its piezoelectric motors, $P_{T}=P_{N}$. The remainder of this section is dedicated to devising virtual fixtures that modify the behavior of the tip $P_{T}$ while using the handle motion $P_{N}$ as the indicator of the operator's intentions.

\section{A. Point Virtual Fixtures}

We begin the formulation of virtual fixtures for Micron by considering the simplest fixture: fixing Micron's tip to a single goal point $P_{G} \in \mathbb{R}^{3}$ in space. While the point virtual fixture is active, the control system should enforce the relationship $P_{T}=P_{G}$, regardless of how the operator moves Micron's handle. We define the very simple control law:

$$
P_{T}=P_{G}
$$

and use approximated linear inverse kinematics with a PID controller and a notch filter at the manipulator resonance to 
drive the tip to the goal position. Controller latency is $\sim 3 \mathrm{~ms}$ and settling time is $<200 \mathrm{~ms}$ if the goal point is within Micron's current range of motion. Once the point virtual fixture is activated, Micron uses the PID controller to maintain the tip on the goal at a control frequency of $1 \mathrm{kHz}$. Moving the virtual fixture, for instance in response to shifting anatomy, is possible by setting a new goal point $P_{G}$. To avoid high-frequency oscillations caused by PID overshoot, large changes in $P_{G}$ should be smoothed with either a low-pass filter or a minimum jerk trajectory.

\section{B. Higher-Order Subspace Virtual Fixtures}

Higher order subspaces can be built on top of the point virtual fixture to obtain more interesting behaviors. Each additional level adds a degree of freedom to the tip motion. For instance, the point virtual fixture restricts all motion. However, a line virtual fixture allows the tip to freely travel along a line while restricting motion orthogonal to the line. Likewise, we can define the hierarchy of virtual fixtures as point, curve, surface, and volume (see Figure 3). To reconnect the need for these virtual fixtures back to medical applicability, it is worth considering possible medical relevance for each virtual fixture:

- $\quad$ Point (0 DOF): Steadying cannula during injection [14]

- Curve (1 DOF): Following path for laser oblation, guiding suture/needle along a blood vessel

- Surface (2 DOF): Maintaining a constant standoff distance, navigating in narrow crevices

- Volume (3 DOF): Restricting the tip volumetrically to prevent tissue contact outside of "safe" areas [11]

Each virtual fixture in the hierarchy collapses to a special case of the one below it. For instance, a line virtual fixture can be implemented with a point virtual fixture that moves alone the line. In fact, we assert that all virtual fixtures can be implemented easily with just the point virtual fixture.

The key to implementing higher-order fixtures with point fixtures is selecting the right point on the higher-order virtual fixture. Algebraically, any point on a fixture will satisfy the constraint imposed by the fixture. Geometrically, it is most intuitive to select a point on the virtual fixture as close as possible to the operator's intended position. Thus for an arbitrary virtual fixture $V$, the optimal goal position $P_{G}$ is the orthogonal projection of $P_{N}$ onto the virtual fixture
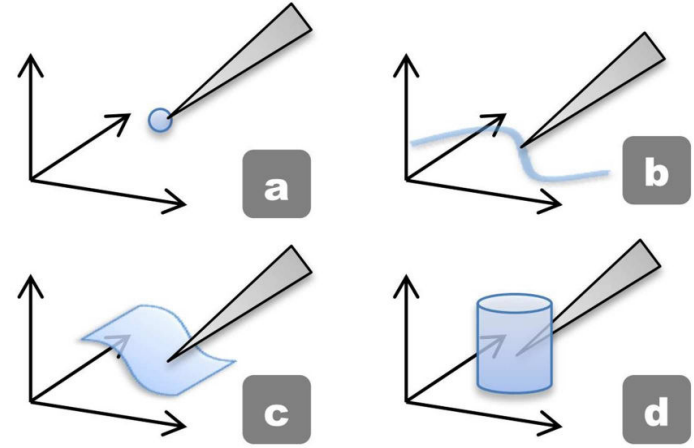

Fig. 3. Virtual fixtures constraining the tip to a subspace with increasing degrees of freedom: (a) point, (b) curve, (c) surface, and (d) volume.
$V$. We define this orthogonal projection by the mapping $\boldsymbol{M}_{\boldsymbol{O}}: \mathbb{R}^{3} \rightarrow \mathbb{R}^{3}$ that selects the goal point $P_{G}$ as the closest point from the null position $P_{N}$ to the virtual fixture $V$ :

$$
P_{G}=M_{\boldsymbol{o}}\left(V, P_{N}\right)
$$

For the simple geometric structures used in this paper (lines, planes, circles, cylinders, etc.), analytic solutions for $\boldsymbol{M}_{\boldsymbol{O}}$ exist. For curves and surfaces described by more complex models such as NURBS, interested readers should look to numerical methods [15].

\section{Tremor Suppression}

In point virtual fixtures, all degrees of freedom are proscribed by the fixture. However, for higher-order virtual fixtures, tremor parallel to the subspace is not affected by the orthogonal projection $\boldsymbol{M}_{\boldsymbol{o}}$. In fact, tremor is evident in all 3 DOF for volume virtual fixtures. One easy solution is to insert a tremor suppression filter $\boldsymbol{F}_{T}^{\boldsymbol{n}}: \mathbb{R}^{\boldsymbol{n}} \rightarrow \mathbb{R}^{\boldsymbol{n}} n \in\{1,2,3\}$. For instance, we can suppress tremor on the $3 \mathrm{D}$ null position $P_{N}$ before it is orthogonally projected on the fixture:

$$
P_{G}=\boldsymbol{M}_{\boldsymbol{o}}\left(V, \boldsymbol{F}_{\boldsymbol{T}}^{3}\left(P_{N}\right)\right)
$$

Alternatively, the tip position can be re-parameterizing on the subspace represented by the virtual fixture with a lower degree of freedom. In this case, we redefine the mapping $\boldsymbol{M}_{\boldsymbol{o}}^{\boldsymbol{n}}: \mathbb{R}^{3} \rightarrow \mathbb{R}^{n}$ as transforming $P_{N}$ in $3 \mathrm{D}$ space to a $n \mathrm{D}$ reparameterized goal position on the virtual fixture via orthogonal projection. For instance, $P_{G}$ on a curve can be reparameterized by arc length and filtered with a single dimensional tremor suppression filter $\boldsymbol{F}_{\boldsymbol{T}}^{\mathbf{1}}$. For an $n$ DOF virtual fixture, we can calculate the goal point as:

$$
P_{G}=\boldsymbol{F}_{\boldsymbol{T}}^{\boldsymbol{n}}\left(\boldsymbol{M}_{\boldsymbol{o}}^{\boldsymbol{n}}\left(V, P_{N}\right)\right)
$$

For experiments in this paper, we use Eq. 3 for volume and plane virtual fixtures and Eq. 4 for curves to prevent any nonlinearities in the orthogonal projection from affecting the tremor suppression. It is also important to note that noise in virtual fixture placement can inject high-frequency movement similar to tremor. Therefore, if virtual fixtures are being generated by video, high-frequency components should be eliminated with a low-pass or Kalman filter.

\section{Motion Scaling}

So far we have described hard fixtures where the tip position cannot deviate from the constraint imposed by the virtual fixture. However, most virtual fixture derivations introduce soft virtual fixtures that share control between the virtual fixture and the operator. If hard virtual fixtures are analogous to an unyielding metal ruler, then soft virtual fixtures can be likened to a yielding rubber ruler that helps draw straight lines, but can be partially overridden by the operator. An additional parameter $\lambda \in[0,1]$ defines how much the operator can override the virtual fixture. In our formulation, $\lambda$ represents the proportion of the hand motion $P_{N}$ vs. the goal point $P_{G}$ that Micron actuates to the tip: 


$$
P_{T}=(1-\lambda) P_{G}+\lambda P_{N}
$$

In essence, $\lambda$ functions as a weighted average of the goal and null position. $\lambda=0$ corresponds to a hard virtual fixture, and $\lambda=1$ disables virtual fixtures entirely. However, for values of $\lambda$ between 0 and 1 , Eq. 5 can be directly manipulated into a motion scaling paradigm:

$$
\begin{gathered}
P_{T}=P_{G}+\lambda\left(P_{N}-P_{G}\right) \\
e=P_{N}-P_{G} \\
P_{T}=P_{G}+\lambda e
\end{gathered}
$$

Thus, the parameter $\lambda$ is seen to be the scaling factor on the error $e$ between the hand position $P_{N}$ and the goal point $P_{G}$ on the virtual fixture. For example, if $\lambda=1 / 2$, then all hand motions that deviate from the virtual fixture will be scaled by one half.

\section{E. Generalized Control Law}

To summarize the control law that incorporates virtual fixtures, tremor suppression, and motion scaling into the Micron framework, we assume for simplicity's sake that tremor suppression on the null position is sufficient and reparameterization is not necessary. Assuming all operations are in 3D, the generalized control law then becomes:

$$
\begin{gathered}
P_{G}=\boldsymbol{M}_{\boldsymbol{o}}\left(V, \boldsymbol{F}_{\boldsymbol{T}}\left(P_{N}\right)\right) \\
e=\boldsymbol{F}_{\boldsymbol{T}}\left(P_{N}\right)-P_{G} \\
P_{T}=P_{G}+\lambda e
\end{gathered}
$$

Eq. 9 selects the goal point on the virtual fixture closest to the tremor suppressed null position. Eq. 10 then calculates the error between these two points. Finally, Eq. 11 drives the tip to either the virtual fixture or, if $\lambda$ is non-zero, scales the error to achieve motion scaling about the virtual fixture.

\section{F. Combining Virtual Fixtures}

The primitive virtual fixtures of point, curve, surface, and volume can be easily combined into compound virtual fixtures. In Section IV, we demonstrate a box virtual fixture built from four plane segments. One difficulty encountered when combining virtual fixtures occurs when the mapping $\boldsymbol{M}_{\boldsymbol{O}}$ becomes degenerate. For instance, when two lines or planes form a right angle, the null position $P_{N}$ might be positioned equidistant between both virtual fixtures. One solution to prevent the tip from oscillating between virtual fixtures is to disable all fixtures except the current one until the tip nears the edge. Only when the tip reaches the boundary between fixtures can Micron transition from one virtual fixture to another. This approach allows the construction of stable compound virtual fixtures.

\section{G. Vision System: Generating Fixtures}

So far, the discussion of virtual fixtures has assumed the availability of each fixture. In reality, virtual fixtures are

likely to be generated from anatomical structures perceived by the vision system. Our setup uses stereo cameras attached to the microscope operating at $54 \mathrm{~Hz}$ with a custom resolution of $504 \times 324$ to track both the instrument tip and the artificial anatomy from which virtual fixtures are generated. For ease of tracking, the Micron Nitinol tip is painted with highly reflective white acrylic paint. Simple image processing techniques including thresholding and blob tracking allow the vision system to accurately locate the instrument tip. The tip in image coordinates is registered to the $2 \mathrm{kHz}$ pose information provided by the ASAP optical trackers as detailed in [13]. To maintain registration, an adaptive least squares routine is run at new camera updates.

For preliminary testing, which is described in detail in Section IV, we use a planar target etched on rubber to generate virtual fixtures (see Figure 4). Hierarchical template matching is used to locate the target. The template matching method is enhanced to handle occlusions of the target caused by the tip of the instrument and its shadow, yielding very fast and robust tracking of the target. Virtual fixtures are derived from the 3D location of the target. While the tracking and virtual fixture formulation presented here could handle moving virtual fixtures, this paper does not explicitly explore that aspect.

\section{EXPERIMENTS AND RESULTS}

Several experiments were performed with Micron to validate the presented virtual fixture formulation.

\section{A. Experimental Procedure}

We use a experimental setup and series of tests similar to [1]. As depicted in Figure 4, virtual fixtures are derived from a target laser etched into rubber. The target includes four crosses arranged as corners of a $600 \mu \mathrm{m}$ square with a 500 $\mu \mathrm{m}$ circle in the middle. Four tasks were considered:

- Hold Still: Hold the tip of the instrument $500 \mu \mathrm{m}$ above the top right cross for $60 \mathrm{~s}$. A point fixture was used.

- Circle Tracing: Trace a circle with a $500 \mu \mathrm{m}$ offset from rubber surface three times. A $3 \mathrm{D}$ curve fixture in the shape of a circle was used.

- Move and Hold: Move the tip to each cross sequentially and hold at each cross for 3 s. Four plane segments oriented vertically and connecting each of the four crosses were used, forming a compound box fixture. Because of the vertical orientation of the planes, the z-movement is unconstrained, allowing the operator

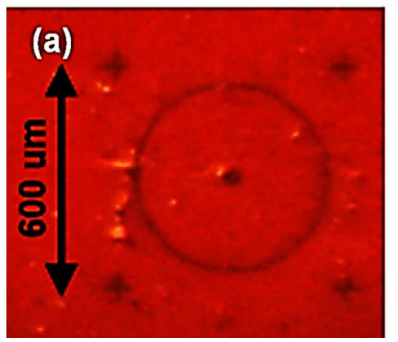

(b)

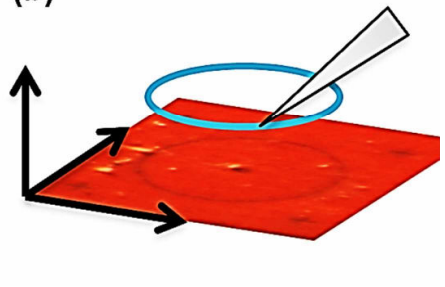

Fig. 4. (a) Laser etched target onto rubber surface (b) Generating a circle virtual fixture from the tracked target. 


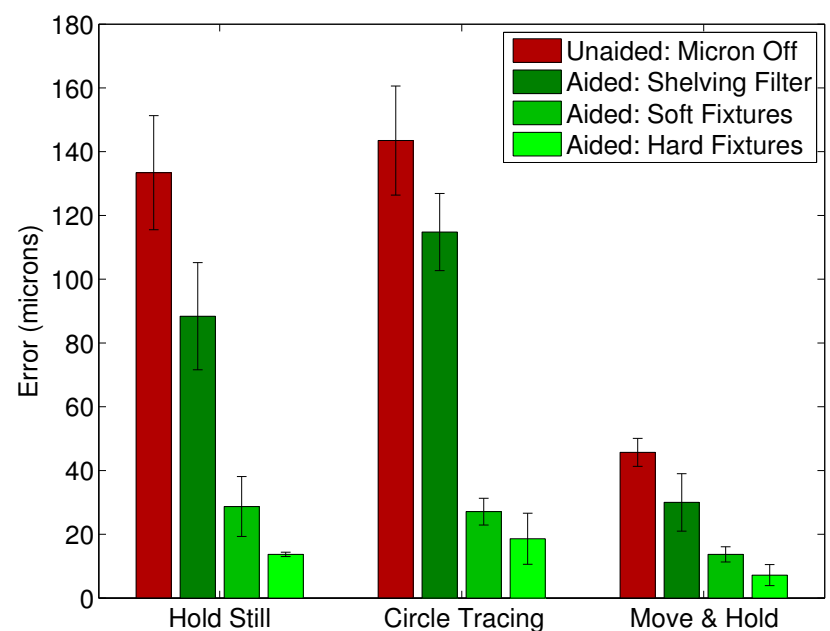

Fig. 5. Mean RMS error across three trials of each combination of task and scenario, with error bars representing the standard deviation.

to choose vertical the offset from the rubber surface.

- Volume Restriction: Allow free movement inside the volume while preventing the tip of the instrument from leaving the volume. A cylindrical volume was used with the circle serving as the perimeter constraint and the rubber surface serving as the bottom constraint.

All experiments were performed by a single individual familiar with Micron but having no surgical experience. Experiments were performed randomly to alleviate ordering effects. Four different scenarios were tested three times:

- Unaided: Micron was turned off.

- Aided with Shelving Filter: Micron was turned on with the state-of-the-art tremor suppression and relative motion scaling algorithm from [1].

- Aided with Soft Virtual Fixtures: Micron was turned on and using virtual fixtures with the motion scaling factor $\lambda=1 / 5$, so errors were reduced by $5 \mathrm{X}$.

- $\quad$ Aided with Hard Virtual Fixtures: Micron was turned on with virtual fixtures but no motion scaling $(\lambda=0)$.

\section{B. Error Measurement and Visualization}

All error was measured as the distance between the tip position measured by the ASAP optical trackers and the closest point on the virtual fixture as measured by the registered stereo cameras. Error was recorded at $\sim 2 \mathrm{kHz}$. Because hard virtual fixturing attempts to remove all error between the tip and the virtual fixture, it is imperative to

TABLE I

MAX ERROR IN POSITIONING TASKS

\begin{tabular}{|c|c|c|c|c|}
\hline \multirow[b]{2}{*}{ T ask } & \multirow{2}{*}{$\begin{array}{l}\text { Un aided } \\
\text { Micron } \\
\text { Off́ }(\mu \mathrm{m})\end{array}$} & \multicolumn{3}{|c|}{ Aided } \\
\hline & & $\begin{array}{c}\text { Shelving } \\
\text { Filter [1] } \\
(\mu \mathrm{m})\end{array}$ & $\begin{array}{c}\text { Soft } \\
\text { Fixtures } \\
(\mu \mathrm{m})\end{array}$ & $\begin{array}{c}\text { Hard } \\
\text { Fixtures } \\
(\mu \mathrm{m})\end{array}$ \\
\hline Hold Still & 489 & 253 & 165 & 67 \\
\hline Circle Tracing & 446 & 312 & 100 & 171 \\
\hline Move \& I Iold & 246 & 172 & 52 & 109 \\
\hline
\end{tabular}

Max error is across all three trials of each combination of task and scenario. a

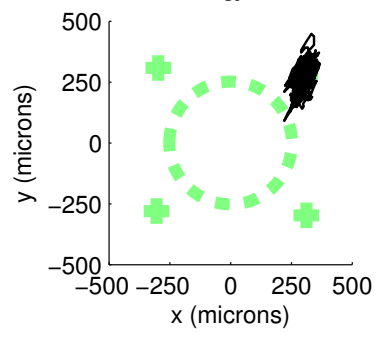

C
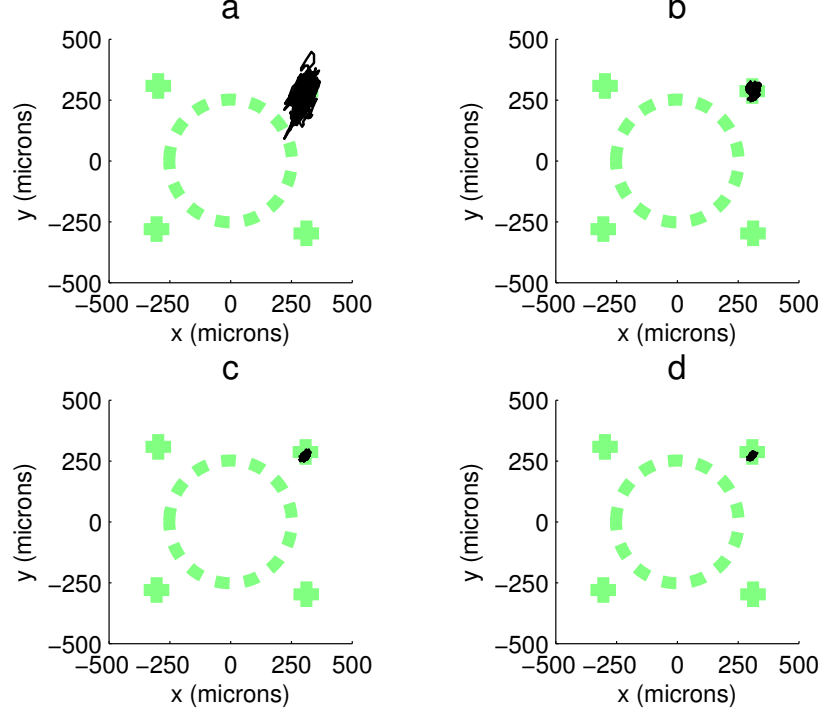

d

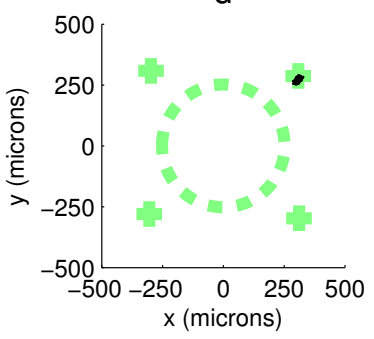

Fig. 6. Hold still results (a) unaided (b) aided with shelving filter [1] (c) aided with soft fixtures (d) aided with hard fixtures.

provide the operator with some visual feedback that lets the operator keep the gross positioning of the handle near the virtual fixture. Otherwise, the operator will unknowingly drift away as the error-eliminating controller disrupts the eye-hand feedback loop. To remedy this, we provide the operator an on-screen visual cue that provides $3 \mathrm{D}$ information as to the location of the unseen null position $P_{N}$. In all tests, the operator used these visual cues to maintain positioning and found the learning curve to be minimal.

\section{Results}

Figure 5 displays mean RMS error with standard deviation across all three trials for each task and scenario combination, demonstrating that virtual fixtures are effective in reducing error. Figures 6-8 show one trial of each task and scenario with a trace of the $2 \mathrm{D}$ tip position overlaid in black on the target represented by dashed light green lines. Maximum error for each set of trials is presented in Table I. It is interesting to note that the Move \& Hold has much lower overall error; since the fixture is not restricting $\mathrm{Z}$ movement, error normally caused by poor depth perception is not evident. In volume restriction tests, hand motion exceeding $500 \mu \mathrm{m}$ was successfully restricted to the volume with a max error of $25 \mu \mathrm{m}$.

\section{DISCUSSION AND FUTURE WORK}

In this paper, we have presented a derivation of virtual fixtures for handheld micromanipulators that depends not on forces applied to a robot arm, but hand motion at the handle of the instrument. The virtual fixtures are generated from stereo cameras in real-time and integrate tremor suppression and motion scaling. Using Micron as a test platform, virtual fixtures have been validated with medically relevant artificial tests and have been shown to outperform state-ofthe-art tremor compensation [1] in reducing the positioning error of the micromanipulator. 


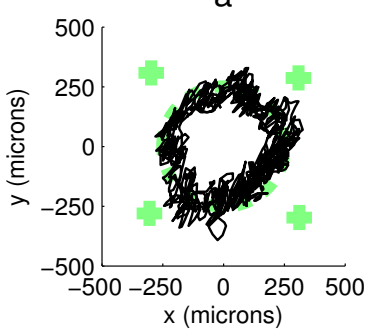

C

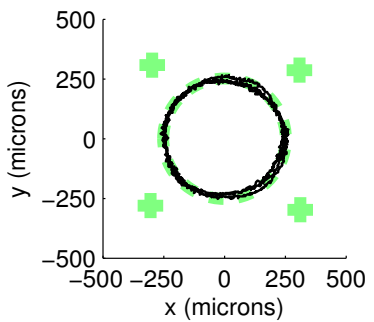

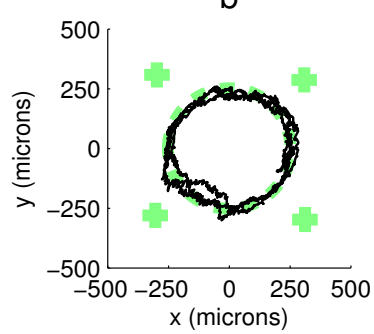

d

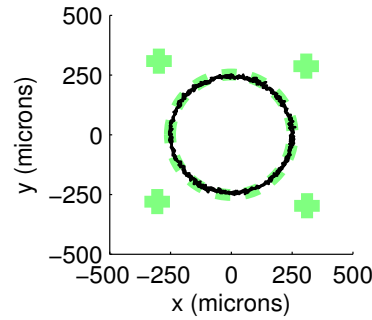

Fig. 7. Circle tracing results (a) unaided (b) aided with shelving filter [1] (c) aided with soft fixtures (d) aided with hard fixtures.

Although virtual fixtures based on the null position of a handheld micromanipulator introduces difficulties relating to degenerate solutions, this formulation offers a number of advantages that simplifies implementation compared to force-based virtual fixtures. No special treatment is needed when maintaining a point virtual fixture as it is in [7]. Implementation of soft virtual fixtures under our formulation directly results in intuitive motion scaling.

In the future, we wish to generalize the formulation of virtual fixtures to parameterizable subspaces and develop better heuristics to deal with degenerate orthogonal projections. Future work will include application of the virtual fixtures to medical procedures such as vitreoretinal microsurgery where extremely high precision is required within ranges of motion capable by Micron. Further testing in more realistic situations is necessary to fully validate the efficacy of the proposed work.

\section{ACKNOWLEDGMENT}

Thanks to Prasanna Velagapudi and Pyry Matikainen for visualization via Plinth (http://code.google.com/p/plinthengine/).

\section{REFERENCES}

[1] J. Cuevas Tabarés, R. A. MacLachlan, C. A. Ettensohn, and C. N. Riviere, "Cell Micromanipulation with an Active Handheld Micromanipulator," in Proc. 32th Annu. Intl. Conf. IEEE Eng. Med. Biol. Soc, 2010, pp. 4363-4366.

[2] S. P. N. Singh and C. N. Riviere, "Physiological tremor amplitude during retinal microsurgery," in Proc IEEE 28th Annual Northeast Bio Eng Conf, 2002, pp. 171-172.

[3] F. Peral-Gutierrez, A. L. Liao, and C. N. Riviere, "Static and dynamic accuracy of vitreoretinal surgeons," in Proc. 26th Annu. Intl. Conf. IEEE Eng. Med. Biol. Soc., 2004, pp. 27342737.

[4] H. Das, H. Zak, J. Johnson, J. Crouch, and D. Frambach, "Evaluation of a telerobotic system to assist surgeons in

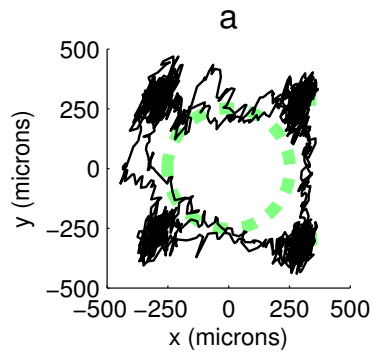

C

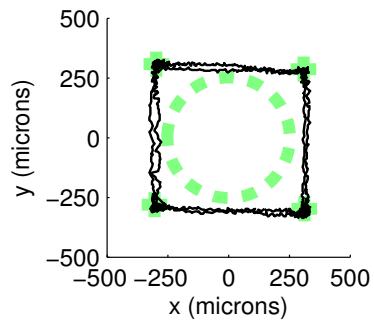

b

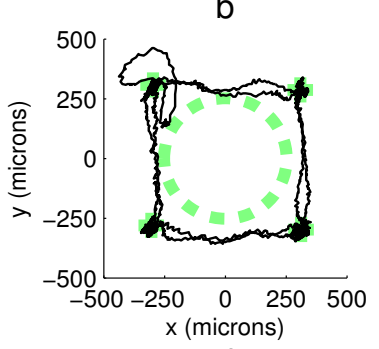

d

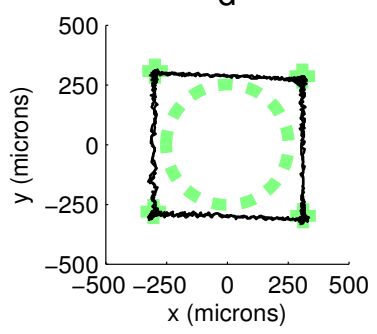

Fig. 8. Move \& hold results (a) unaided (b) aided with shelving filter [1] (c) aided with soft fixtures (d) aided with hard fixtures.

microsurgery," Computer Aided Surgery, vol. 4, pp. 15-25, 1999.

[5] B. Mitchell, J. Koo, M. Iordachita, P. Kazanzides, A. Kapoor, J. Handa, G. Hager, and R. Taylor, "Development and application of a new steady-hand manipulator for retinal surgery," in Proc. IEEE Int. Conf. Robot. Autom., 2007, pp. 623-629.

[6] C. N. Riviere, W. T. Ang, and P. K. Khosla, "Toward active tremor canceling in handheld microsurgical instruments," IEEE Trans. Robot. Autom. , vol. 19, pp. 793-800, 2003.

[7] A. Bettini, P. Marayong, S. Lang, A. M. Okamura, and G. D. Hager, "Vision-assisted control for manipulation using virtual fixtures," IEEE Trans. Robot. , vol. 20, pp. 953-966, 2004.

[8] H. C. Lin, K. Mills, P. Kazanzides, G. D. Hager, P. Marayong, A. M. Okamura, and R. Karam, "Portability and applicability of virtual fixtures across medical and manufacturing tasks," in Proc. IEEE Int. Conf. Robot. Autom, 2006, pp. 225-231.

[9] M. Li, M. Ishii, and R. H. Taylor, "Spatial motion constraints using virtual fixtures generated by anatomy," IEEE Trans. Robot. , vol. 23, pp. 4-19, 2007.

[10] A. Kapoor, M. Li, and R. H. Taylor, "Constrained control for surgical assistant robots," in Proc. IEEE Int. Conf. Robot. Autom, 2006, pp. 231-236.

[11] B. L. Davies, S. J. Harris, W. J. Lin, R. D. Hibberd, R. Middleton, and J. C. Cobb, "Active compliance in robotic surgery - the use of force control as a dynamic constraint," Proc. Inst. Mech. Eng., Part H: J. Eng. Med., vol. 211, pp. 285-292, 1997.

[12] R. A. MacLachlan and C. N. Riviere, "High-speed microscale optical tracking using digital frequency-domain multiplexing," IEEE Trans. Instrum. Meas., vol. 58, pp. 1991-2001, 2009.

[13] B. Becker, S. Voros, R. MacLachlan, G. Hager, and C. Riviere, "Active guidance of a handheld micromanipulator using visual servoing," presented at the Proc. IEEE Int. Conf. Robot. Autom., Kobe, Japan, 2009.

[14] B. C. Becker, S. Voros, J. Louis A. Lobes, J. T. Handa, G. D. Hager, and C. N. Riviere, "Retinal vessel cannulation with an image-guided handheld robot," in Proc. 32th Annu. Intl. Conf. IEEE Eng. Med. Biol. Soc, 2010, pp. 5420-5423.

[15] Y. L. Ma and W. T. Hewitt, "Point inversion and projection for NURBS curve and surface: control polygon approach," Computer Aided Geometric Design, vol. 20, pp. 79-99, 2003. 